\title{
Public health ophthalmology
}

\author{
CARL KUPFER
}

From the National Eye Institute, National Institutes of Health, Bethesda, Maryland 20892, USA

SUMmary Public health ophthalmology, often referred to as preventive or community ophthalmology, focuses on three community based activities: preventive, curative, and promotive. The pioneering work of Professor Barrie Jones in this field is recognised. Examples of each activity are provided, with emphasis on the developing world.

Professor Barrie Jones can be considered one of the pioneers in the field of public health ophthalmology, with particular emphasis on the integration of eye care into the health care delivery systems in the countries of the developing world. His blending of research activities both in the laboratory and in the field with the excellent public health ophthalmology course in the Department of Preventive Ophthalmology in London has resulted in a career replete with scientific achievements as well as training a new generation of participants dedicated to carrying on his work in many parts of the world.

I found it most interesting to review his bibliography, which revealed a broad spectrum of interests ranging from longstanding activity in research dealing with chlamydial infections in general and trachoma in particular, corneal graft surgery, the aetiology and chemotheraphy of herpes simplex infection, and pharmacological studies of antiviral and antionchocerciasis drugs, to name only a few. But I was most impressed with his abiding and continuing interest in veterinary medicine appearing in the New Zealand Veterinary Journal and other similar publications dealing with such diverse subjects as idiopathic, familial hyperchylomicronaemia in a cat, adamantinoma in a cat, feline leukaemia virus testing, a collagen dysplasia in a greyhound bitch, gastric carcinoma in a bitch, and myotonia in related chow chow dogs. This broad spectrum of interests demonstrates not only his research virtuosity but also a commitment to report interesting and important research observations whenever and wherever they may occur.

Preventive, or community, or public health ophthalmology has only recently begun to attract attention in the ophthalmological world. There are three activities that constitute this approach to the Correspondence to Carl Kupfer, MD. delivery of eye care, namely, preventive, curative, and promotive activities.

\section{Prevention of disease}

Preventive activities rely heavily on research, not only performed in the laboratory but also carried out in the community by epidemiological techniques to identify high risk populations for specific diseases. The cohort studies conducted in Framingham, Massachusetts, helped to establish smoking, systemic hypertension, overweight, and lack of physical exercise as risk factors for the development of coronary heart disease. The numerous case control studies in both Great Britain and the United States established cigarette smoking as the major risk factor for lung cancer. To perform these studies we must leave our office and proceed to the community to design the study, recruit the participants, and conduct the examinations. In the developing world, especially South-east Asia, risk factor assessment in preventing the increasing morbidity and mortality from blinding malnutrition secondary to vitamin A deficiency has pointed to infant diarrhoea, measles infection, and borderline liver stores of vitamin $A$ as probable risk factors. In each case prompt and adequate administration of vitamin $\mathrm{A}$-in oral rehydration fluids in the case of diarrhoea or by mouth in oil suspension - can protect the child from developing clinical signs and symptoms of xerophthalmia and keratomalacia. By identifying those populations at highest risk, the distribution of vitamin A can be targeted primarily to those children in greatest need of it.

A second example of preventive activity deals with identifying those individuals in populations at greatest risk for developing angle-closure glaucoma. We know that in China, and countries to the south 
including Laos, Vietnam, Cambodia, and extending to Burma, angle-closure glaucoma is much more prevalent than open-angle glaucoma. In fact it has been estimated that $50-90 \%$ of all glaucoma in these areas is of the angle-closure type. Thus a screening outreach programme to identify those individuals over the age of 35 years whose anterior chamber depth is $1.7 \mathrm{~mm}$ or less would identify such a high risk population. If such a population was followed up for five years and the incidence of angle-closure glaucoma attacks was $5 \%$ per year or more, then one could consider prophylactic iridotomy to prevent such an occurrence. There is now available a portable, battery operated YAG laser which could be used in the field to perform an iridotomy under topical anaesthesia. With such an outreach programme, especially in those countries where the major population still resides in rural communities, a serious cause of blindness and visual disability could be prevented.

\section{Curative programmes}

The second component of public health ophthalmology is curative. In the developing world, cataract is much the most prevalent cause of blindness and offers an excellent example of the curative aspects of public health ophthalmology by a well proved, safe, and effective surgical intervention. Such a curative programme can function most effectively if it combines an efficient screening programme to identify people blind from cataract, a strategy to make the surgery available and to motivate the patient to seek such treatment, and a facility whereby the surgical procedure and postoperative care can be provided. Where possible, such curative components should be integrated into the existing health care infrastructure, so that screening for cataract can be performed by primary health care personnel in villages and small towns and the surgery performed in rural districts or provincial hospitals with appropriate facilities and personnel. The great challenge facing our profession is to increase the efficiency of cataract surgery without decreasing its safety. In the developing world alone there are about 14 million blind from cataract and perhaps as many as five million new cases each year. Thus the total number of operations performed each year in the developing world must be trebled, from a present level of $2 \frac{1}{2} / 2$ million to at least seven million both to keep up with new cases and to reduce the backlog of cataract blind. It is apparent from the formula below that, to increase the cases operated per year,

$$
\text { Cases/year }=\frac{\text { number of beds } \times \text { days/bed available/year }}{\text { length of stay/days }}
$$

one must either increase the number of beds, which is very expensive, increase the number of days that the beds are made available, which is already at a maximal number, or decrease the length of postoperative stay in hospital. At present the average length of stay is about five to six days. If this could be reduced to one or two days, a three-fold increase in capacity to perform cataract surgery would be possible. This increased capacity must be coupled with increased case finding and increased motivation on the part of the patient to seek surgery.

\section{Education}

The third component to be discussed is the promotive component. Educational material can provide simple ways of improving personal hygiene, advise on proper diet such as adding dark green leafy vegetables high in beta-carotene to the diet of young children, and increase awareness of the availability of surgery to restore sight to the cataract blind. Legislative action can be directed towards better safety conditions in mining operations or supporting training programmes for allied health personal, who play such an important part in delivering eye care within the whole health care system. Publicity directed towards shaping public opinion on reducing the prevalence of blindness can be as effective in changing the behaviour of individuals and achieving a 'health revolution' as it was in shaping the behaviour of farmers in India with respect to the 'green revolution'. All audiovisual techniques available can be harnessed to this end.

Finally increased co-operation and collaboration among the national committees of each country, the active non-governmental organisations that are sponsoring ongoing programmes, and the country representatives of the World Health Organisation will help mobilise needed resources and enhance political will for there to be a strong and effective prevention of blindness programme within the overall health care national programme. Only then, in the words of Professor Jones, will the 'burden of avoidable blindness' be removed once and for all time. 